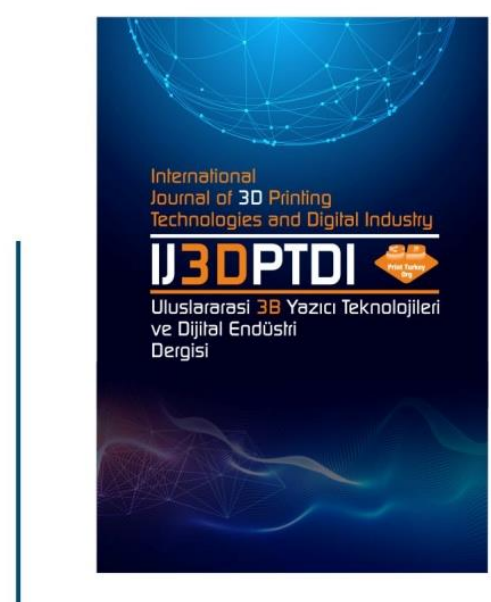

ULUSLARARASI 3B YAZICI TEKNOLOJILERI

VE DIJITAL ENDÜSTRI DERGISI

INTERNATIONAL JOURNAL QF 30 PRINTING TECHNOLOGIES AND DIGITAL INDUSTRY

IS5N:2602-3350 [Online]

URL: https://dergipark.org.tr/ij3dptdi

\title{
TROUBLESHOOTING AND TACKLING THE COMMON PROBLEMS IN VAT PHOTOPOLYMERIZATION AND FDM 3D PRINTING
}

Yazarlar (Authors): Joonas Kortelainen(D), Mirka Leino*(D), Tommi Lehtinen(D)

Bu makaleye şu şekilde atıfta bulunabilirsiniz (To cite to this article): Kortelainen J., Leino M., Lehtinen T" Troubleshooting And Tackling The Common Problems In Vat Photopolymerization And FDM 3d Printing" Int. J. of 3D Printing Tech. Dig. Ind., 5(2): 281292, (2021). 


\title{
TROUBLESHOOTING AND TACKLING THE COMMON PROBLEMS IN VAT PHOTOPOLYMERIZATION AND FDM 3D PRINTING
}

\author{
Joonas Kortelainen $^{\text {a }}$ (D), Mirka Leino ${ }^{a^{*} \text { (D) }}$, Tommi Lehtinen ${ }^{\text {a }}$ iD \\ a Satakunta University of Applied Sciences, Faculty of Technology, FINLAND \\ * Corresponding Author: mirka.leino@samk.fi
}

(Received: 20.06.2021; Revised: 16.07.2021; Accepted: 20.08.2021)

\begin{abstract}
The importance of 3D printing as a builder of the digital future is growing all the time. When it comes to the digital future, one clear goal is that, for example, spare parts are no longer necessarily shipped from factories around the world, but are 3D printed using a digital model of the spare part purchased from the manufacturer. Using 3D printers for replacement printing or home use is relatively simple. However, with 3D printers, you come across a wide variety of situations that require fault detection, troubleshooting, and general adjustment. This article highlights the most common problems with 3D printers. The technologies chosen for this study are Vat Photopolymerization and FDM (Fused Deposition Modeling) printing technologies because they are very common techniques for spare parts printing and home use. This study was conducted in concrete way, experimenting and producing problems intentionally, then solving them with solutions and techniques, which are common and gained through experience. As a result, this article is written as a guide to the most common problems with these chosen technologies. Solutions to the problems are showcased in simple and effective steps. After all, this study showed well the diversity of problem situations in 3D printing as well as the need for concrete expertise in troubleshooting solutions. The problems faced and tackled in this study were the most common ones but there can exist other problems too.
\end{abstract}

Keywords: Vat Photopolymerization. FDM. Common problems. Troubleshooting.

\section{INTRODUCTION}

The importance of 3D printing as a builder of the digital future is growing all the time. When it comes to the digital future, one clear goal is that, for example, spare parts are no longer necessarily shipped from factories around the world, but are 3D printed using a digital model of the spare part purchased from the manufacturer. This digital approach not only ensures faster availability of spare parts, but also allows products and parts to be personalized in almost any way.

When 3D printing is used on a small scale to print spare parts needed for company's own production processes or for personal use at home, printers are often not the most expensive on the market and therefore certainly not the highest quality. However, 3D printers, which are often intended for smallscale use, are of sufficient quality for their intended use. Using these 3D printers for replacement printing or home use is relatively simple. However, with 3D printers, you come across a wide variety of situations that require fault detection, troubleshooting, and general adjustment.

This study examines the most typical problems of consumer 3D printers and their solutions using case study methods. The research focuses on the challenges and problems in Vat Photopolymerization and FDM printing technologies as well as presents their most common solutions. Problems and fault situations are treated as concrete cases and solutions to them are structured on a theoretical basis, but especially through concrete experiments and analyzes. The results of the cases are presented as identified problems and fault situations combined with case-specific solutions. In this way, combining theoretical knowledge and practical experiments will find solutions to the real situations of users using small-scale $3 \mathrm{D}$ printing. 


\section{RESEARCH FRAMEWORK AND METHODOLOGY}

The framework of this study consists of 3D printing equipment for consumer use and their general operating conditions both in home use and in companies printing for their everyday spare part needs. This research is fundamentally based on the constructive research approach. It has influenced the outline of the research but also all the individual case studies of the common problems with 3D printers. This research approach is based on a clear tendency to produce new information based on experimentation and testing, but at the same time based on theoretical knowledge. In accordance with constructivism, special attention is paid to linking and explaining empirical findings from the perspective of theory. The purpose of this research is to perform experimental 3D printing tests and to examine possible problems that arise in them. Through this research, better practices for 3D printing are refined according to a constructive research approach [1-3].

The practical research phase of this study is carried out using the methods of the case study, because separate experimental 3D printing tests are carried out following the steps of the case study research method. Case study is a good experimental method for this research work, because the cases to be studied are very individual and each of them is studied from its own specific perspective. However, in some cases, several cause-and-effect relationships are combined. According to the case study method, the cases of this research are closely connected to the known theories and through them analyzes and conclusions are formed. This research is in line with the case study method also from the point of view that the processing of data is largely based on observations made on the basis of the cases. The development cases have been selected very appropriately and no random sampling is used. The selection of cases has been made based on their prevalence. Goals for this research were set in the beginning and the research plan was drawn up based on the goals, but according to the case study method, the plan can be changed if the cases require so. In this way, the research plan took shape in its final form during the research $[4,5]$.

In accordance with the constructive research approach and case study method, the plan for this research is as follows:

- $\quad$ Selecting the 3D printing methods to be studied

- $\quad$ Studying the theories of selected 3D printing methods

- $\quad$ Selecting the 3D printing cases to be examined

- $\quad$ Experimenting and analyzing cases

- $\quad$ Reflecting cases and their results in theory

- Drawing conclusions

The research plan is based on the research question with the aim of identifying the problems and fault situations of the selected 3D printing methods and their solutions.

\section{EXPERIMENTAL RESEARCH}

This study focuses on identifying the most typical problems and failure situations of FDM and MSLA printers (Masked Stereolithography, which is one way to do 3D printing based on vat photopolymerization) and finding solutions to them through experimental operations based on the case study. Problems and fault situations were produced appropriately in order to catch their origin and solution as practically as possible. The 3D printing methods, FDM and MSLA, are chosen for this research because of their largest user base. Their popularity among home users and companies doing small-scale printing is largely based on the low cost, ease of use, and wide availability of the devices.

This study was picked also because 3D printing itself has risen up to be such an easily approachable technology. Because the ease of use of 3D printing it has spread to almost every area of society, it pushed us to work on this study, to provide concrete examples and solutions of 3D printing troubleshooting [6]. There are only few books and articles available on the topic, and most of them are from around 20102016, thus the need was seen for an updated study with modern printers and technologies. Especially since MSLA is a new technology, and only recently accessible with a low cost $[7,8]$. 


\subsection{Challenges and problems of FDM printers and their solutions}

In this chapter the challenges of different FDM methods and common problems or failure situations are studied. Problems and fault situations have been treated as separate cases and solutions to these problems and fault situations have been compiled in connection with them. FDM printer problems can be divided into four categories: user errors, device errors, material errors, and slicing software errors. On the other hand, the error of the slicing program is an error made by the user, so one could probably say that there are three categories of error.

Then considering the common problems with 3D printers, it is important to keep in mind that the most common problems can be avoided by taking good care of the printer. It is a good idea to perform various inspections of the printer, and especially its mechanics, from time to time. At least the mobility of the bearings, the tension and movement of the belts and the cleanliness of the trapezoidal screws should be checked. The general cleanliness of the printer environment should be taken care. There should be no sawdust, metal dust, or the like in the room, which could cause problems for the printer's mechanics [6][7].

\subsubsection{Case 1: Adhesion of the print to the print bed}

The most important thing when 3D printing is the adhesion of the print to the print bed. Of course, the adhesion of the first print layer to the print bed is the most important. From the first layer you can see whether the print will succeed or fail after a few layers. Many failed prints may be caused by the poor first layer adhesion (Figure 1b), which has then detached the print from the print bed in the final stages. The tests showed that at worst, poor adhesion in the print can cause the print to detach slightly from the print bed and the printer to continue printing so that the plastic sticks to the nozzle itself (Figure 1a). The print tests performed in this study and the deliberate adhesion problems caused in them indicated that the adhesion problems may have several different reasons, or the reason may be the sum of several reasons. The most common reasons were:

$$
\begin{aligned}
& \text { - } \quad \text { Wrong nozzle distance from the print bed } \\
& \text { - } \quad \text { The print bed is not horizontal } \\
& \text { - } \quad \text { Incorrect print temperature } \\
& \text { - } \quad \text { Dirty print bed } \\
& \text { Prints adhere too tightly on the print bed }
\end{aligned}
$$

\section{Wrong nozzle distance from the print bed}

The tests proved that the wrong distance of the nozzle from the print bed can be seen from the fact that the extruded plastic is very round, and when touched with a finger, it immediately detaches from the print bed (Figures 1c and 1d). The optimal height for the nozzle from the print bed would be when the plastic is flattened slightly but not too much, as this will reduce print dimensional accuracy on the lower part. If, on the other hand, the plastic does not flatten enough, the adhesion of the print and the interlayer bonding suffer, which can lead to poor print durability. Recalibrating the Z-height for the printer is a simple solution to this. It is recommended to recalibrate either in the printer settings, or by physically moving the Z-axis limit switch closer to the print bed, or by lowering / raising the print bed with the print bed screws.

\section{The print bed is not horizontal}

If the print bed is not horizontal that can be seen during the first layer of printing, especially on larger prints, one edge is more flat or high than the other edge. The print bed can be adjusted simply by using the bed adjusting screws. At this point, it is important that the printer is turned off and the nozzle is gently moved by hand. The adjustments can be done by using an A4 paper that is transported from one corner of the printer to another while moving the nozzle to the same corners. The gap between the nozzle and the print bed should be such that the nozzle clearly hits the paper, but the paper moves in the gap between the nozzle and the print bed. Finally, make sure the adjustments are made at all corners and in the center of the print bed. If the printer does not have adjusting screws, adjustments must be made programmatically using the so-called virtual surface adjustment of edges. 


\section{First layer print speed too high}

Too high print speed for the first layer is noticed because the print stays poorly on the print bed during the first layer, even if the print height is set to optimal. Experiments showed that the printer feeder does not keep up with the speed of the printer nozzle. In this case, the printer can't extrude enough material to protrude evenly between the nozzle and the print bed, so it would stick to the print bed. The tests showed that the print speed of the first layer should be reduced so that it is no more than half of the basic print speed of the printer. This can be done in the slicing program, e.g., under "Initial Layer Speed". It is a good idea to initially set the speed value to around $30 \mathrm{~mm} / \mathrm{s}$, this varies by the printer. By changing and experimenting with this, a value at which the output no longer sticks to the print bed will be found. At the experimental part of the study the printing temperature was also tried to increase in order to improve the adhesion on the first layer. At higher temperatures, the feeder mechanism must do less work to extrude the material, thus overcoming the problem of too little material. However, based on the experiments, it can be said that a better option is to lower the print speed of the first layer.

\section{Incorrect print temperature}

The adhesion of the 3D printed part is a complicated issue. Once the printer speed and height have been optimized, but the 3D printed part still does not stick to the print bed, it is time to consider other options, like print temperature. Too low printing temperature will make it difficult for the feeder to operate and may prevent the material from sticking to the bed. Certain materials, on the other hand, require a temperature within very well-defined limits in order to adhere to the bed. The study also confirmed knowledge that every material manufacturer as well as material have specific printing temperatures. If the print temperatures recommended by the material or material manufacturer are known, they are placed in the slicing program settings. Based on the experiments, other recommended modifications are at least a +10 degrees increase in nozzle temperature. Modification experiments can be done more carefully, i.e. the above-mentioned temperature is first increased by 5 degrees. A useful way to determine the optimal printing temperatures on a particular material is to print a so called "Temperature Tower" (Figure 1e). Temperature Tower is a 3D printed part in which the same shape is printed on top of each other at different temperatures. It is used to find the optimal temperature for the layer thickness and material used.

\section{Dirty print bed}

When all the above settings were restored, print adhesion problems were caused by dirt on the print bed. A greasy print bed greatly affects the adhesion of prints. This is best noticeable in the glass and mirror print beds. In the tests, the print bed was soiled and cleaned with various substances. Based on the tests, it is a good idea to clean the glass and mirror bed, for example, with a degreasing dishwashing detergent from time to time if there are problems with adhesion. It is also important to rinse the print bed and dry it well with a paper towel after washing but avoid touching the print bed. Then, for example, if the print bed is Polyetherimide (PEI) or similar, it is a good to clean it with isopropyl alcohol and paper.

\section{Prints adhere too tightly on the print bed}

Next, a situation was tested in which the 3D printed part adheres too tightly to the print bed. In this case, removing the $3 \mathrm{D}$ printed part requires mechanical aids that run the risk of parts coming off the print bed. According to the tests, too good adhesion can be caused by at least a few reasons: the print nozzle is too close to the print bed, the bed temperature is too high, or the extrusion multiplier set in the software is too high, meaning the feeder pushes too much material when printing. Some materials, such as PETG and various flexible materials, sometimes adhere too tightly to the print bed. Too tight adhesion can be prevented by pretty much the same steps as adhering too loosely. First, checking the height of the print head so that it does not print too close to the print bed. If materials other than PLA are used, such as PETG or flexible materials, it is a good idea to set the distance of the printhead from the print bed slightly higher than normal, as these materials require a slightly different pressure between the nozzle tip and the print bed. The experiments showed that in this situation, it is also a good idea to check the extrusion multiplier of the material used in the printing software. The printer may extrude too much material into the $3 \mathrm{D}$ printed part and cause an adhesion problem, but it can also cause variations in the appearance of the $3 \mathrm{D}$ printed part. 


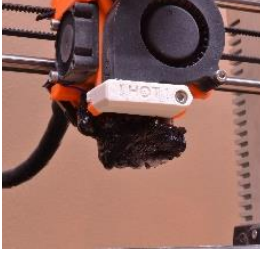

(a)

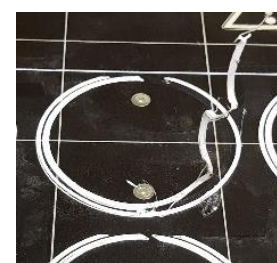

(b)

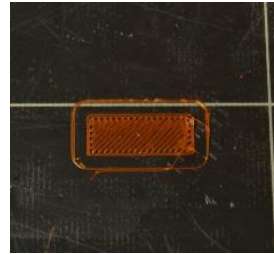

(c)

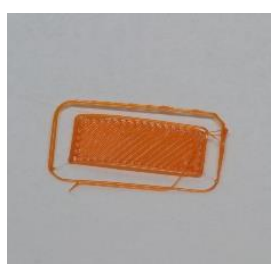

(d)

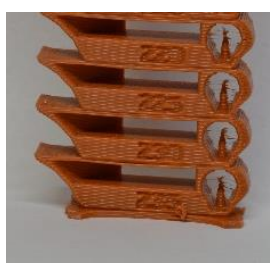

(e)

Figure 1. Multiple images of bad print bed adhesion $(a)(b)(c)(d)$ and image of temperature tower (e).

\subsubsection{Case 2: The print risen from one corner}

Based on the tests, it was found that there are a few factors that can affect the print so that it starts to rise from one corner up in the middle of printing and starts to warp. Distortion occurs most with large prints, especially when printing with ABS plastic, for example. Tests showed that this was due to excessive temperature differences between the 3D printed part, the surrounding room, and the print bed. When printing, the plastic expands slightly but begins to shrink as it cools. If the temperature difference is too large and cooling is too rapid, detachment may occur. Some materials appear to be more susceptible to such detachment than others. The most sensitive are, for example, ABS and Polycarbonate. A solution to this problem is sought from the solutions in the previous section, but also by adjusting the temperature of the printing room and, in particular, by preventing strong airflows. The room temperature should not be very low, so heating the room or the printing chamber can be a good solution. Also, the temperature of the print bed should be checked. It is good to have approx. $+90-+95$ ${ }^{\circ} \mathrm{C}$ with ABS plastic. Another important thing to check is the cooling of the part produced by the printer, which should only start after printing several layers. For non-PLA plastics, cooling should not even be used at full capacity. Third solution to the printing problem is to turn on adhesion helper settings on the slicing software. In this case, it would be a good idea to turn on the "Brim" setting. The tests showed that the width of the Brim setting should be extended to improve the adhesion of the print to the bed. A fourth solution to the warping problem is the use of various adhesion aids. These include hairspray, ABS slurry, various adhesion sprays developed for 3D printing and ordinary craft glue. All of these should be used with caution and only when the print bed is not warm. However, the spreadability of the glue stick improves when the print bed is warm, so it can be applied when the print bed is warm.

\subsubsection{Case 3: Holes and missing parts in the 3D printed part}

Sometimes there are small holes in the 3D printed part, or walls with holes in the structure. In this case, the durability of the print is poor even if a more durable material is used. Then, if the print is twisted or torn, it will break up between layers. According to the tests, this is often because the adhesion between the layers is too weak, the adhesion of the walls is poor, or the print is simply missing material. Figure 2 shows the situation when the internal filling is incomplete. Tests showed several reasons for such a print:

- $\quad$ The printer nozzle may be clogged or have a small blockage.

- $\quad$ The print speed is too high and the print head cannot keep up with the extrusion speed required by the printer.

- $\quad$ Bowden-style printers may have a worn Bowden tube.

- $\quad$ The printing temperature might be too low.

- $\quad$ There may be dirt in the printer's feed mechanism.

- $\quad$ The diameter of the printing filament is not even.

- $\quad$ The spool of the printing material does not rotate freely.

- $\quad$ The printing material might have absorbed some moisture.

Nozzle clogging will be addressed more extensively later and excessive printing speed have been addressed before.

\section{Bowden-style printers may have a worn Bowden tube}

Long-term tests showed that Bowden tubes could wear out or debris could be formed inside. Usually this was due to the left-over plastic entering the tube from the extrusion mechanism. The simplest solution to this seems to be to clean the tube every now and then. The tube is removed and blown with 
compressed air or it is washed with water and dried well. Some tests also showed that even this did not help, so the end solution was just to replace the whole tube.

\section{The printing temperature might be too low}

Tests showed that the printing temperature was often something completely different from what the manufacturer had stated. For example, in a situation where the printer nozzle temperature is $+210{ }^{\circ} \mathrm{C}$ according to the control panel, but normal PLA does not pass through the nozzle. A couple of different reasons for this were found in the tests: 1. in a user-built printer, the problem may be in the firmware configuration, if the thermistor type is selected incorrectly, the printer will display completely incorrect temperature values, 2. the material of the nozzle requires higher temperatures, for example, the thermal conductivity of a nozzle made of stainless steel or hardened steel is very different from that of a nozzle made of brass and they require higher temperatures for printing. The tests showed that this kind of temperature problems can be solved by using PID tuning for the nozzle and a silicone sock to stabilize the temperature on the heat block.

\section{There may be dirt in the printer's feed mechanism}

Sometimes the printer has difficulty extruding material because there is a clog in the nozzle. The tests showed how in this situation the extrusion mechanism continues to extrude the material, causing the extruder gears that push the filament into the extruder to gradually strip the filament and form small pieces of plastic and plastic dust. The plastic pieces and dust then fill the gaps between the teeth of the gears, so that the gears no longer grip to the filament. The gears can be easily cleaned mechanically by a toothbrush.

\section{The diameter of the printing filament is not even}

Manufacturing tolerances or what is promised by different material manufacturers range from $+/-0,02$ $\mathrm{mm}$ to $+/-0,07 \mathrm{~mm}$. Based on long-term tests, this can be classified as a rare problem, but it does occur. This is more typical for cheaper filament manufacturers, as they might cut some corners in the quality control of the materials. Tests with different materials have although shown that price for the material does not guarantee quality every time. The solution to the problem is to measure the diameter from different spots of the material spool and input the average value into the slicing software.

\section{The spool of the printing material does not rotate freely}

If the printer seems not to cause the printing problem, it is important to check the external factors that affect printing. For example, if the filament spool cannot rotate freely, the power of the extruder mechanism may not be sufficient to pull the filament off the roll. According to the tests, the most common cause of excessive force required to rotate a filament spool is a knot in the filament. The knot may have been generated by a user error or already in the filament spooling process. The best way to prevent the formation of knots is to ensure that when the spool is transported, the filament cannot swing freely, but is attached to a hole in the spool for this purpose.

\section{The printing material might have absorbed some moisture}

Some print materials are more hygroscopic than others, such as polycarbonate and nylon. Hydroscopicity is a problem especially when materials are not stored properly. The tests found that if the material absorbs moisture, its printability deteriorates considerably, and the extrusion result becomes very uneven and "zitted". Hydroscopic materials should always be stored in a closed container, including during printing. However, if the material absorbs moisture, it can be dried by heating, e.g. in an oven, for several hours. The oven temperature and drying time depend on the material. 


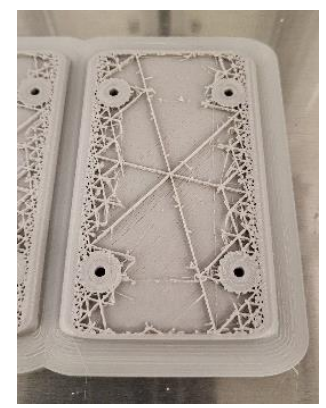

Figure 2. 3D print with incomplete filling.

\subsubsection{Case 4: The outer dimensions of the 3D printed part inaccurate}

The higher the dimensional accuracy of the 3D printed parts, the more likely there are situations in which the outer dimensions of the 3D printed part are inaccurate. The tests proved clearly that usually it is a challenge to get the outer dimensions just right. According to the tests, there are several different reasons for the variation in the dimensions. With a self-assembled printer with a user-configured firmware, the most common reason emerges in the extruder stepper motor settings. Another significant reason seems to be the variable behavior of materials. Materials with different densities act differently when extruded through the nozzle. Some materials, like silk filament, expand once they are extruded through the nozzle. One solution to the inaccurate outer dimensions is to calibrate the stepper motor steps accordingly. The required steps per mm (in $\mathrm{X}, \mathrm{Y}, \mathrm{Z}$ directions) can be calculated through different calculators, but the extruder stepper motor needs to be calibrated through a few steps: 1 . material is inserted normally to the printer and stopped once it starts coming out of the nozzle, 2. ca. $120 \mathrm{~mm}$ of the material is measured and marked before the extruder, 3 . the printer is made to extrude $100 \mathrm{~mm}$ of the material, 4. the distance between the mark and the extruder is measured and if it's $20 \mathrm{~mm}$, the stepper motor is all calibrated. Otherwise, the new steps required for the stepper motor should be calculated. This can be done by the following formula:

$$
\text { New value }(\text { Steps per } m m)=\text { Previous value }(\text { Steps per } m m) \times \frac{\text { Desired distance }(\mathrm{mm})}{\text { Measured distance }(\mathrm{mm})}
$$

Once the new value is set in the configuration, the calibration steps should be ran again to be sure that the calibration is successful.

Once the stepper motor has been calibrated but the inaccuracy persists, a material calibration must be performed. The density of the material affects how thick the line of material is extruded. For calibration, a test cube with known dimensions and two outer walls, without top surfaces and without infill is printed (Figure 3). The thickness of the walls of the printed cube is measured and if the measured thickness is to two times the diameter of the nozzle, the material is calibrated. If the measured value is more than $0,04 \mathrm{~mm}$ above the desired value, the extrusion multiplier must be reduced. If it's under the desired value, then the extrusion multiplier needs to be raised. This should be repeated until the desired tolerance is achieved.

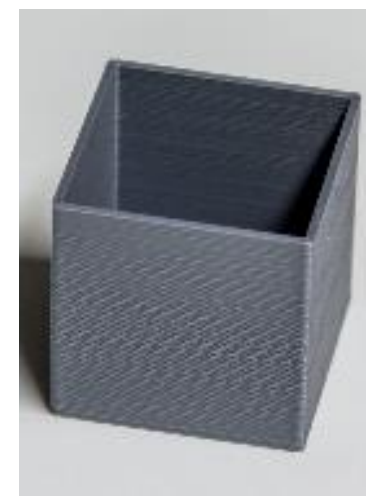

Figure 3. Example of a 3D printed calibration cube. 


\subsubsection{Case 5: Print head clogged}

The tests revealed situations where, at the end of a large print run, the nozzle hovers a few centimetres above the print and does not extrude (Figure 4a). This means that the nozzle is clogged or there is a problem with the extruder. Some of the causes and solutions to this problem have already been addressed in Case 3, but there are a few other causes and solutions, especially related to the extrusion mechanism and the print head. If the motor of the extruder mechanism makes a clicking sound and the motor rotates clockwise for a few rounds and then suddenly returns counterclockwise, there is too high resistance in the extrusion mechanism to push the filament. This may be because the gears of the extrusion mechanism bite too hard into the filament (Figure 4b). On the other hand, the nozzle temperature may be too low to prevent the filament from melting. This can be solved by loosening the gears and raising the nozzle temperature. Another cause may be that the ambient temperature is too high. The tests showed that for example when printing a PLA filament at an ambient temperature of $+40^{\circ} \mathrm{C}$, the extruder motor will start clicking, because the plastic has melted or softened causing a clog inside the nozzle.

The solution is to cool down the environment and electronics. If cooling of the environment is not possible, it is advisable to switch to another material. For example, PETG does not suffer from too high ambient temperature. Also, a printer where different types of material are repeatedly printed, might suffer from debris or left over filament from other materials, and that can cause a clog. Solution for this is to do a so-called cold pull for the print head, which clears the nozzle from the debris (Figure 4c).

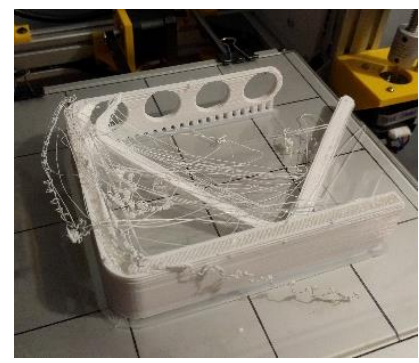

(a)

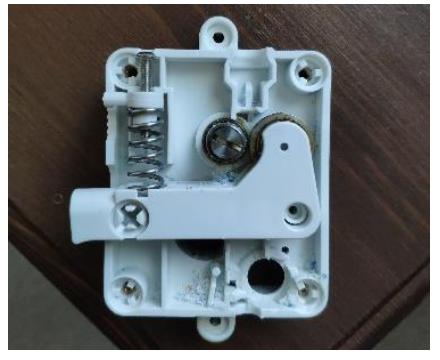

(b)

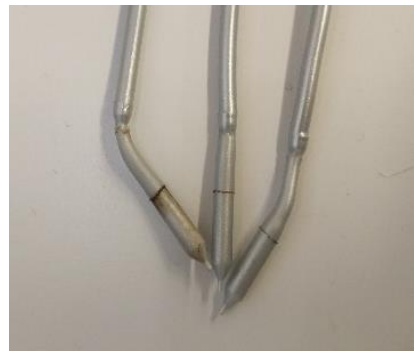

(c)

Figure 4. Material stopped extruding while printing (a), gears grinding the filament (b), debris from nozzle (c).

\subsubsection{Case 6: Molten plastic on top of the heat block leaks onto the print}

Leaking material on top of the print head (Figure 5), or on top of the heat block, can cause that material leaks also onto the print. This may cause deformations or quality deviations on the print, or even print failure due to a sudden blob of molten plastic appearing on the surface and the printer head hitting that during printing movements. The reason for this situation is the poor attachment of the throat above the heat block or the lack of post-tightening, leaving a cavity between the heat block and the throat through which the material travels over the heat block. This can be fixed by disassembling the print head and tightening the parts while they are heated up. This can be done when the nozzle temperature has reached at least $+100{ }^{\circ} \mathrm{C}$.

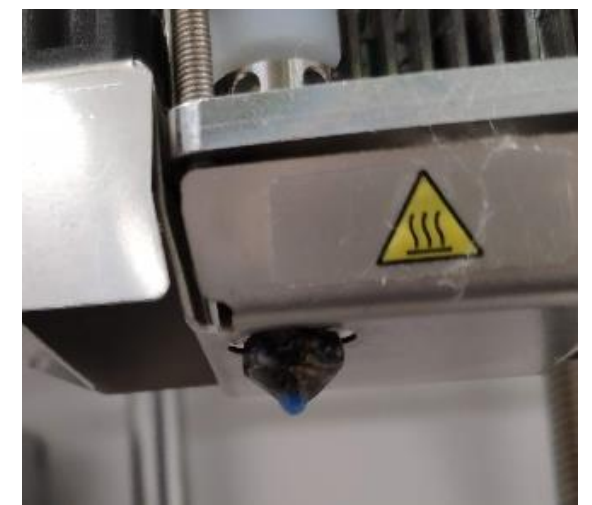

Figure 5. Molten plastic leaking from top of the heat block. 


\subsubsection{Case 7: Printer starts a new layer slightly on the side}

The tests showed that sometimes the printer starts a new layer a bit from the side compared to the previous layer (Figure 6a). According to the tests, the layer shift, can be due to a few different reasons:

- $\quad$ X or Y pulley screws are loose

- $\quad$ Printer belts are loose or too tight

- $\quad$ Stepper motor driver overheats or doesn't give enough current

\section{$\mathrm{X}$ or Y pulley screws are loose}

In case of the pulley screw being loose (Figure 6b), it was noticed that even though the motor turned, the pulley itself didn't turn accordingly, and thus the belt won't move at all. When the printers are used for several hundreds or thousands of hours, the screws should be tightened from time to time.

\section{Printer belts are loose or too tight}

One common reason for skipping steps for the motors, or layer shifts, can be the printer belt. If the belt is too loose, the pulley gear will not get a grip, so it won't move it properly. On the other hand, if the belt is too tight, the motor and motor driver need to work harder to get the belt moving. The solution is simply to tighten or loosen the belt.

\section{Stepper motor driver overheats or doesn't give enough current}

Based on the tests, the stepper motor driver can also cause problems. The stepper motor driver can give too much current to the motor and therefore overheat. The solution to this is to install cooling for the stepper motor drivers, for example a simple fan blowing on the stepper motor driver helps quite a lot, as usually the drivers have heatsinks already installed. Another way is to start adjusting the current that is driven to the motor drivers. This can be done through firmware configuration or measuring Vref voltage on the stepper motor driver and adjusting the potentiometer accordingly.

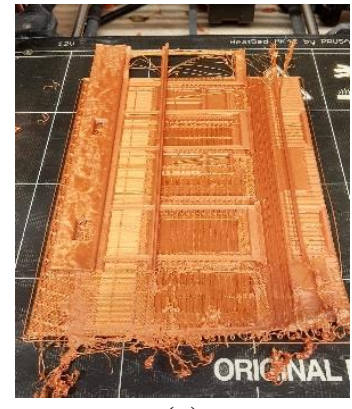

(a)

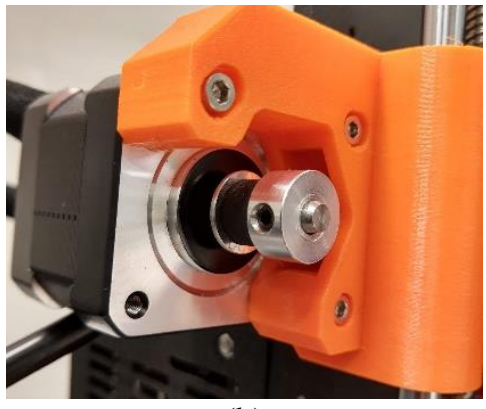

(b)

Figure 6. Image of a layer shift (a) and of a loose pulley screw (b).

\subsection{CHALLENGES AND PROBLEMS OF MSLA PRINTERS AND THEIR SOLUTIONS}

The problems with MSLA printers are quite different from those with FDM printers, mainly because MSLA printers do not have as many moving parts. Usually, the causes of the problems are due to a couple of factors. However, problems with MSLA printers are not so easy to fix without the cost to the user. All parts for MSLA printers are quite expensive compared to parts for FDM printers. The most common consumables for MSLA printers are FEP (Fluorinated ethylene propylene) film and an LCD (Liquid-Crystal Display). Based on the tests, it was found that the FEP film should be replaced when its surface starts to be very worn. The LCD screen is usually damaged due to heating. It is difficult to estimate the time of replacement because the service life of LCD monitors varies greatly. The need for replacement is identified when printing.

\subsubsection{Case 8: The print does not stick to the build platform}

Sometimes when the print is completed and the print bed is raised, there is nothing on the build platform. In this case, the entire printing has taken place in the resin tank, and nothing has stuck to the print platform. In this case, it is advisable to empty the resin tank through a filter back into the resin bottle 
and clean it with warm water or isopropyl alcohol. Based on the tests, from the print adhesion point of view, the adhesion of the first layer is a critical step. There may be a few different reasons for the failure of the first layer to adhere:

- Build platform not leveled against the LCD

- Print settings not optimized for the resin in use

- The resin tank screws not tightened properly

\section{Build platform not leveled against the LCD}

The tests found that if the printer's build platform does not lower enough to touch the FEP film, the first layer of the print has no chance of adhering to the build platform. For this reason, it is important to calibrate the $\mathrm{Z}$ axis whenever any changes are made, or it is suspected that the build platform has skewed. This is basically done the same for every MSLA printer, by unscrewing the tightening screws on the build platform and lowering that onto the LCD. It is recommended to use a plain A4 paper between the build platform and the LCD just to indicate the distance between these two and to protect the LCD from scratching (Figure 7).
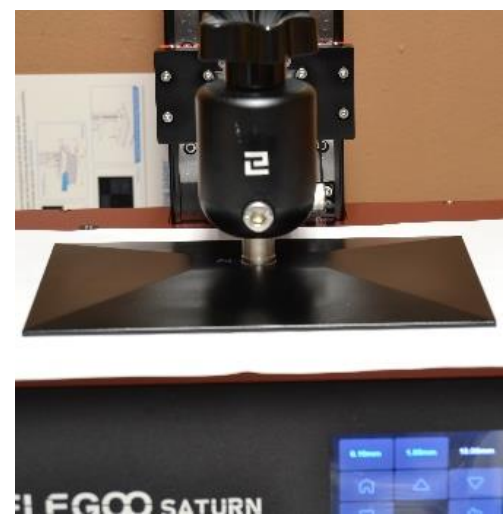

Figure 7. Using a plain A4 paper between the LCD and the build platform.

\section{Print settings not optimized for the resin in use}

The tests showed that resin cure times vary, so the exposure time of the certain resin being used should always be considered when printing with an MSLA printer. The exposure time needs to be adjusted for each printer individually. If the exposure time is too short, the resin will not cure properly and the print will fail, while if the exposure time is too long, the cure will be too high, and details will disappear from the print. Exposure time calibration is necessary with both a new resin and a new printer. The exposure time should be figured out through by trial and error. For some printer models, there is also a calibration print file created by the printing community that allows one to test different exposure time on the same print run. The printed calibration print can be used to visually decide, which exposure time to use. There are also community created test tables for each type of resin and color available for free use, from which a reference values can be selected for the used materials.

\section{The resin tank screws not tightened properly}

According to the tests, poor print stability can be also caused by a human error. If the resin tank screws are not tightened after replacing or cleaning the tank, the print may no longer adhere to the build platform. The same problem can arise as well with build platform's screws. These problems can be easily avoided by making a list for the steps for each phase of the printing, thus these steps could become a routine for the user.

\subsubsection{Case 9: Faulty FEP film}

A faulty FEP film is one obvious reason for MSLA printing failure. Based on the tests, visual inspection may not be the best way to determine the condition of the FEP film, as dents and scratch looking marks may not affect the function of the film, but the elasticity of the film is a significant factor. For this reason, the condition of the film should be viewed by tapping it with a finger. If tapping the film gives out a nice sound, like a drumstick hitting a drum, the film is fine, but if it sounds more like a thud, like rock 
dropping on the floor, then it's not tight enough and causes failing prints. Tightening the film does not seem to be a possible move, so the only working solution to this is to change the FEP film. A useful aid in installing the film, according to the test, is a block slightly higher than the resin tank, which allows the film to be tensioned in place.

\subsubsection{Case 10: The base of the print remains on the build platform while the supported part of the print comes off}

When the MSLA printer finishes a layer, it needs to peel the print off from the FEP film. At that moment there's a small force pulling the part, and if the used supports aren't thick or dense enough, the print may drop from the build platform into the resin tank. According to the tests, this phenomenon became particularly apparent when printing large models, as the size, number and shape of the supports relative to the size of the model are lesser. These problems can be prevented by orienting the part so that it's always a bit angled without large flat surfaces against the build platform (Figure 8), and hollowing large models, thus reducing their weight. On the other hand, the thickness, density and shape of the supports can be changed.

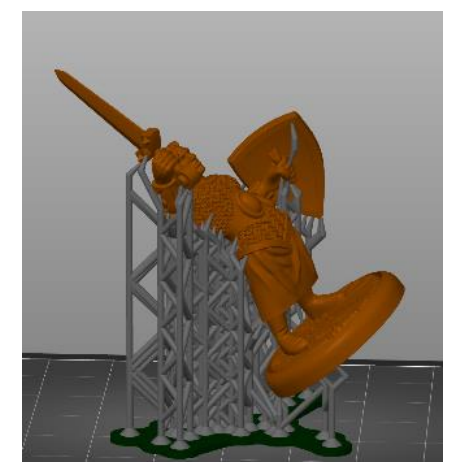

Figure 8. Orienting the part so that there are no large flat surfaces.

\section{DISCUSSION}

This article focused on the troubleshooting of the most common problems with Vat Photopolymerization and FDM (Fused Deposition Modeling) 3D printers. These technologies were chosen for this study because of their very common use for spare parts printing and home use. In order to study the most common problems and find solutions the study was conducted in concrete way. Initially, the most common problems were identified, after which they were researched by causing problems intentionally. Solutions were sought through experience, practical experimentation, and trial and error.

From the beginning, it was clear that many of the problems are ones that many printer users must constantly tackle. The aim of the study was to find the clearest possible methods for solving the problems. The study layout concluded to research seven common problems with FDM printers as well as three common problems with MSLA printers. The aim was to find the simplest and most costeffective methods possible for small-scale users. We believe that such research from the perspective of small-scale printers provides added value through structured problem solving and clear descriptions.

The research is clearly limited to these ten generally identified problems. In the future we consider it important that other problems are identified and their solutions are approached in the same structured way. It is also important to pay attention to the presented solutions in the future in order to find possible new solutions and thereby improve the guidelines developed in this study.

\section{CONCLUSION}

The future of 3D printing is truly positive. Printing technologies are evolving all the time, and as all patents for different technologies are about to expire, or have already expired, it enables even small actors to experiment. Until a few years ago, it was not even possible to think of getting a 3D printer for home use for less than $500 €$, but today one can get it for less than $200 €$. Not to mention MSLA printers, which were worth of several thousand a few years back and are now available for less than $200 €$. Now that the patents for metal printers have expired, they are also starting to appear on the market to an 
increasing extent, even at a price that even smaller machine shops, for example, could consider using them.

Today, printers are manufactured and used in very different conditions and situations, so there are also a lot of problems with them. As the fundamentals of technology begin to become common knowledge, it's time to learn how to troubleshoot and tackle the problems that come with small-scale use. Problems are challenging to do artificially, but they occur in everyday use. This article was written as a guide to the most common problems with Vat Photopolymerization and FDM printing technologies. Solutions to the problems were presented in simple and effective steps. The study demonstrated the diversity of problem situations in 3D printing as well as the need for concrete expertise in troubleshooting solutions. The problems tackled in this study were the most common ones but there can exist other problems too.

This study combined the problems encountered in the daily use of several different printers and their solutions, as well as the artificially caused situations and their solutions. Most of the problems presented are certainly familiar to many users of FDM and MSLA printers, but in this study, the cases were systematically addressed with emphasis on similarities and specialties. Most of the problems and challenges are caused by user-errors or due to printer wear but identifying and recording them as a whole was seen as an important issue in this study. This study was not a complete set of problems with printers but highlighted common problems that clearly prevent printing and their solutions.

\section{REFERENCES}

1. Lukka, K., "Konstruktiivinen tutkimusote", [Constructive research approach] [article in Finnish], https://metodix.fi/2014/05/19/lukka-konstruktiivinen-tutkimusote/, April 1, 2021.

2. Lehtiranta, L., Junnonen, J.-M., Kärnä, S., Pekuri L., "The Constructive Research Approach: Problem Solving for Complex Projects", In Pasian B. editor, Designs, Methods and Practices for Research of Project Management, Pages 95-106, Gower Publishing Limited, Aldershot, 2015.

3. Olkkonen, T., “Johdatus teollisuustalouden tutkimustyöhön”, Report, [Introduction to industrial economics research] [report in Finnish], Helsinki University of Technology, Helsinki, 1993.

4. Aaltio-Marjosola, I., "Casetutkimus metodisena lähestymistapana", [Case study as a methodological approach] [article in Finnish], https://metodix.fi/2014/05/19/aaltio-marjosola-casetutkimus/, April 2, 2021.

5. Yin, R., "Case Study Research: Design and Methods”, 3rd ed., Sage Publications, London, 2003.

6. Bell, C., "Maintaining and Troubleshooting Your 3D Printer", 1st ed., Pages 3-6, Apress L. P., New York, 2014.

7. Horvath, J., Cameron, R., “A Guide to Modeling, Printing, and Prototyping”, 2nd ed., Pages 3-18, Apress L. P., New York, 2020.

8. Griffey, J., “The Types of 3-D Printing”, Library Technology Reports, Vol. 50, Issue 1, Pages 8-12, 2014. 